Zur Bestimmung des Bleies auf maassanalytischem Wege. Yon

\title{
W. Diehl.
}

In Hüttenlaboratorien tritt an den Chemiker oft die Aufgabe heran eine grosse Anzahl armer Bleierze auf nassem Wege auf ihren Gehalt an Blei zu prüfen. Da der bisher eingeschlagene Weg der gewichtsanalytischen Bestimmung als Bleisulfat zu zeitraubend ist und die bis jetzt in Vorschlag gekommenen maassanalytischen Methoden manches zu wünschen übrig lassen, so bemühte ich mich eine bessere zu ermitteln, und es gelang mir durch eine Modification des Verfahrens von $\mathrm{H}$. Schwar $Z^{*}$ ) den Zweck zu erreichen. Das neue Verfahren entspricht seinem Zwecke in sehr befriedigender Weise und bediene ich mich desselben schon seit längerer Zeit mit bestem Erfolge.

Meine Methode beruht auf der Ausfällung des Bleies aus essigsaurer Lösung mit saurem chromsaurem Kali und auf Bestimmung des Chromsäure-Ueberschusses mit unterschwefligsaurem Natron in saurer Lösung.

Ich bediene mich einer $1 / 20$-Normal-Kaliumbichromatlösung, $7,38 \mathrm{~g}$ im Liter enthaltend, von der jeder Cubikcentimeter $0,01035 \mathrm{~g}$ Blei entspricht, und einer Lösung von Natriumhyposulfit, etwa $4^{\circ}-5 \mathrm{~g}$ im Liter enthaltend. Man beginnt damit die Relationen zwischen Kaliumbichromat und Natriumbyposulfit $\mathrm{zu}$ bestimmen. $\mathrm{Zu}$ diesem Zwecke lässt man $20-30 c c$ ersterer Lösung in einen Kolben laufen, verdünnt mit $300 c c$ Wasser und setzt $20-25 \mathrm{cc}$ verdünnte Schwefelsäure (1 Thl. $\mathrm{HO}, \mathrm{SO}_{3}$ und 2 Thle. HO) zu. Ein gewisser Ueberschuss von Schwefelsäure ist unbedingt erforderlich (Salzsäure eignet sich nicht so gut). Ich habe denselben genau festgestellt und obiges Verhältniss lässt den Zweck am besten erreichen. Man kocht nun die Flüssigkeit und lässt tropfenweise von der Lösung des unterschwefligsauren Natrons zufliessen; die Lösung wird immer heller; gegen Ende lässt man nach Zusatz einiger Tropfen immer noch einmal aufwallen. Der Endpunkt ist in der Regel an vollständiger Farblosigkeit der Flüssigkeit zu erkennen und tritt durch einen Tropfen des Reagens' ein. Die Beurtheilung der Flüssigkeitsfärbung wird erleichtert, wemn man den Kolben gegen Ende in eine Porzellanschale stellt.

*) Ding1er's polyt. Journ. 169, 284; diese Zeitschrift 2, 378. 
Xur bei grösseren Mengen chromsauren Kalis tritt keine vollstänđlige Farblosigkeit, sondern eine schwach grünliche Färbung ein. Der Reductionsprocess wird durch folgende Gleichung dargestellt:

$$
\begin{gathered}
4\left(\mathrm{KO}_{2} 2 \mathrm{CrO}_{3}\right)+3\left(\mathrm{NaO}, \mathrm{S}_{2} \mathrm{O}_{2}\right)+13\left(\mathrm{HO}, \mathrm{SO}_{3}\right)=4\left(\mathrm{KO}, \mathrm{SO}_{3}\right) \\
+4\left(\mathrm{Cr}_{2} \mathrm{O}_{3}, 3 \mathrm{SO}_{3}\right)+3\left(\mathrm{NaO}_{3}, \mathrm{SO}_{3}\right)+13 \mathrm{HO} .
\end{gathered}
$$

Will man Erze auf diesem Wege auf Blei prüfen, so schliesst man sie mit Königswasser und verdünnter Schwefelsäure auf, concentrirt durch Abdampfen bis die Schwefelsäure sich zu verflüchtigen beginnt, verdünıt mit Wasser, kocht auf um das schwefelsaure Eisenoxyd in Lösung zu bringen, lässt erkalten und filtrirt durch ein glattes Filter ab, welches man mit schwefelsüurehaltigem Wasser answäscht. Zu dem Rückstand im Kolben (man bringe so wenig als möglich davon auf's Filter) fügt man $15 \mathrm{cc}$ einer Lösung von neutralem essigsaurem Ammon, verdünnt mit etwa $50 \mathrm{ce}$ Wasser, kocht, filtrirt dann durch dasselbe Filter, in velches man einen Tropfen Ammon gebracht hat, in einen unterstehenden Kolben, wiederholt diese Manipulation mit 5 cc essigsaurem Ammon und wäscht schliesslich gut mit kochendem Wasser, dem man etwas letzteren Salzes zugesetzt hat, aus. Cutes Auswaschen ist hierbei Erforderniss, da erfahrungsmässig die Filter die essigsauren und weinsauren AmmonBleisalze mit einer gewissen Festigkeit zurückhalten. Zweckmässig ist es daher das ausgewaschene Filter noch rom Rande aus mit etwas kochender verdünnter Salzsüure (1 Salzsäure von 1,12 spec. G.: 10 Wasser) zu behandeln und mit heissem Wasser nachzuwaschen. Bei dieser Behandlung bleibt dann keine Spur von Bleisalz im Filter zurück. Man wende ausgewaschenes, dünnes Filtrirpapier an. Die erhaltene Lösung des Bleisulfates in essigsaurem Ammon säuert man mit 5-10 Tropfen Essigsäure an und titrirt in der Kälte mit Kaliumbichromat; in der Wärme würde Ammoniumacetat etwas Bleichromat lösen. Bei gutem Schütteln setzt sich der Niederschlag leicht ab, und kann man den Endpunlit bis auf $0,2-0,4 c c$ treffen.

Man gibt indess einen Ueberschuss von mindestens $2 c c$ der ChromatLösung zu um der Löslichkeit des Bleisalzes vorzubeugen. Zweckmässig ist es diesen Ueberschuss bei allen Versuchen möglichst gleich zu nelmen. Nachdem man kräftig umgeschüttelt hat, lässt man eine halbe Stunde stehen und filtrirt. Etwaigem trübem Hindurchgehen begegnet man durch Hinzufügen einiger Tropfen einer Lösung von essigsaurem Natron, welche man mit Essigsäure angesäuert hat. Ist dennoch etwas chromsaures Bleioxyd durch's Filter gegangen, so filtrirt man, wenn 
308 Diehl: Zur Bestimmung des Bleies auf maassanalytisehem Wexe.

die Menge desselten relativ gross ist, noch einmal, kleine Mengen schaden wanig. Das Resultał wird dadurch um ein Geringes zu niedrig. Am besten bewirkt man die Filtration durch zwei in einander gelegte Faltenfilter. Den Niederschlag wäscht man viermal mit kaltem Wasser ans und titrirt die Lösung nach Ansäuern mit Schwefelsäure in oben angegebener Weise.

Um die Anwendbarkeit der Methode zu prüfen wurden folgende Mengen reinen Bleies abgewogen, in Salpetersäure gelöst, mit Schwefelsüure eingedampft und in angegebener Woise mit Ammonacetat gelöst etc. :
1) $0,1246 g$
2) $0,1058 \ll$
3) $0,1364 \ll$
4) $0,1281 \ll$

Finem Cubikcentimeter der Lösung des sauren chromsauren Kalis e.tsprachen 1,80 ce der Lösung des unterschwefligsauren Natrons.

Es warden gebraucht dem unterschweflig-

saures clurom- unterschweflig- sauren Natron ent- dem Blei ontspresaures Kali: saures Natron: sprechendes saures chendes saures

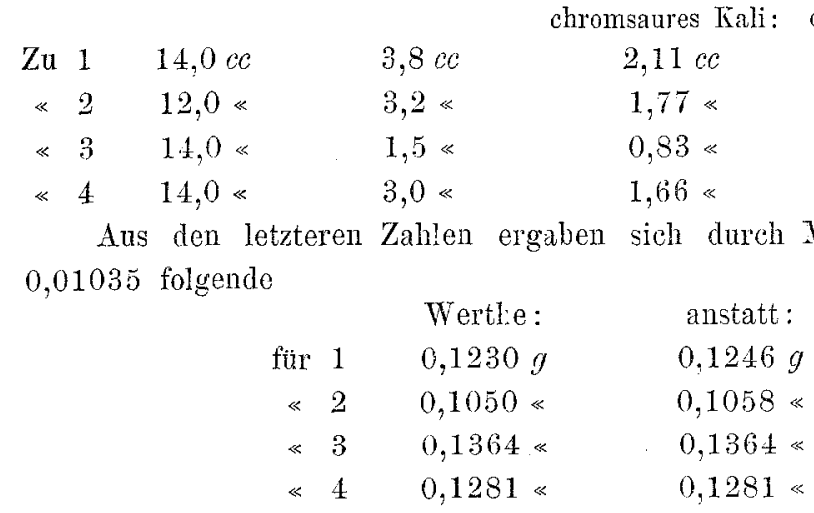

Das Ammoniumacetat verdient vor allen Ammonsalzen zur Lösung des Bleisulfats den Vorzug. Es muss in neutraler oder schwach saurer Lösung angewandt werden um ceine Fähigkeit Bleisulfat zu lösen möglichst auszunutzen. Ammon trübt dicse Lösungen. Ich bediente mich einer durch Neutralisiren gewöhnlichen Ammons mit gebräuchlicher Essigsäure erhaltenen Solution. $1 g$ Bleisulfat bedurfte $15 \mathrm{cc}$ derselben zur Lüsung. 
Meyer: Zurückgehen der eisen- u. thonerdehaltigen Superphosphate. 309

Das weinsaure Ammon ist nicht brauchbar, da es in stark ammoniakalischer Lösung angewandt werden muss, denn freie Weinsäure löst Bleiclromat; beim Ansäuern der ammoniakalischen Lösung fällt aber dann Ammonbitartrat nieder, - und weil Ammontartrat Kaliumbichromat selbst in saurer Lösung reducirt.

Emser Hütte, Februar 1880.

\section{Weitere Hittheilungen über das Znrïckgehen der eisen- und thonerdehaltigen snperphosphate.}

(Berichtigung.)

Von

\section{Dr. Carl Ferd. Neyer.}

Fortgesetzto Untersuchungen über das Zurückgehen der eisen- und thonerdehaltigen Superphosphate haben mir gezeigt, dass die Schlüsse, welche ich aus früher gemachton Beobachtungen gezogen habe, *) falsch sind.

Versetzt man eine concentrirte Lösung von einbasisch phosphorsaurem Kalk **) mit noutralem Eisensulfat, so schcilet sich ein starker Nicderschlag von Gyps aus der. Lösung ab. Dieser Niederschlag bildet sich langsam und besonders wirkt die Gegenwart freier Säuren sehr verhindernd auf die Entstehung desselben ein, so dass bei den ersten Versuchen keine Gyps-Abscheidung von mir beobachtet wurde. Dieser Umstand führte mich zu der Annahme, dass einer Umsetzung zwischen einbasisch phosphorsaurem Kalk und Eisensulfat eine Umwandlung des ersteren in zweibasisch phosphorsauren Kalk, nuter Abspaltung von Phosphorsäure (bewirkt durch Erhitzen oder Verdünnen der Lösung), vorhergehen müsse. Hieraus zog ich die Folgerung, dass in den Superphosphaten der einbasisch phosphorsaure Kalk erst durch Einwirkung auf dreibasisch phosphorsauren Kalk zweibasisches Salz bilde, ehe eine Umsetzung mit dem Eisensulfat eintreten lönne.

*) Diese Zeitschrift 19, 145 .

**) In Betreff der Nomenclatur der Phosphate vergl. die Anmerkung auf S. 145 dieses Bandes. R. F. 